\title{
Teorías subjetivas de niños pertenecientes a Colegios de Coquimbo y La Serena
}

\author{
Lisette Gahona Palominos \\ Universidad de La Serena - La Serena - Chile \\ Jorge Catalán Ahumada \\ Universidad de La Serena - La Serena - Chile
}

\begin{abstract}
Resumen
El objetivo de la investigación ${ }^{1}$ fue analizar y comprender el desarrollo en la actividad explicativa sobre sí mismo y sobre su entorno de 50 niños, entre 6 y 9 años, que cursaban primero básico en dos establecimientos educacionales de La Serena y Coquimbo, Chile, para contribuir a la caracterización de su desarrollo cognitivo. La metodología de estudio fue de tipo cualitativa, privilegiando el uso de técnicas de observación, entrevista semiestructurada y entrevista de grupo focal. Se encontró que los niños elaboran predominantemente teorías sobre elementos de su entorno; que construyen sus teorías basándose en sus experiencias previas, las expresan en forma poco elaborada, pero si se les incentiva a la reflexión pueden dar respuestas de mayor complejidad. Las utilizan para predecir y explicar situaciones, persuadir a las demás personas, como también orientar sus propias acciones, mostrando además una tendencia a corroborar y probar sus teorías.
\end{abstract}

Palabras clave: teorías; cognición; ninõs.

\section{Subjective theories of children from schools of Coquimbo and La Serena}

\begin{abstract}
Abstrac
The objective of the research was to analyze and understand the development in the explanatory activity on itself and its environment 50 children, ages 6 and 9, who were in first two basic schools from La Serena and Coquimbo, Chile, to contribute characterization of cognitive development. The study methodology was qualitative type, favoring the use of techniques of observation, semi-structured interviews and focus group interviews. They found that children predominantly made theories surrounding elements; they build their theories based on their previous experiences, expressed in a sophisticated way, but are encouraged to reflection can give more complex answers. They use them to predict and explain situations, persuade others, as well as guide your own actions, also showing a tendency to check and test their theories.
\end{abstract}

Keywords: theories; cognition; children.

\section{Teorias subjetivas de crianças pertencentes a Colégios de Coquimbo e La Serena}

\section{Resumo}

O objetivo da investigação foi analisar e compreender o desenvolvimento da atividade explicativa sobre si mesmo e sobre seu entorno junto a 50 crianças, entre 6 e 9 anos, que cursavam o ensino fundamental em duas escolas de La Serena e Coquimbo, Chile, de modo a contribuir com a caracterização de seu desenvolvimento cognitivo. A metodologia de estudo foi de tipo qualitativo, privilegiando o uso de técnicas de observação, entrevista semiestruturada e entrevista de grupo focal. Verificamos que as crianças elaboram predominantemente teorias sobre elementos de seu entorno; constroem suas teorias baseando-se em suas experiências prévias, expressam-nas de forma pouco elaborada, mas se são incentivadas a refletir podem dar respostas mais complexas. Utilizam-nas para predizer e explicar situações, persuadir outras pessoas e também orientar suas próprias ações, mostrando também uma tendência a corroborar e provar suas teorias.

Palavras-chave: teorias; cognição, crianças.

1 Investigación enmarcada dentro del proyecto Fondecyt "Asombros educativos infantiles y propensión a aprender", que se encuentra en ejecución desde el año 2011. 


\section{Introducción}

El estudio de la cognición y el aprendizaje infantil implica conocer la forma en que los niños organizan sus conocimientos sobre el mundo, el cómo construyen categorías de la realidad, las estrategias de aprendizaje que utilizan, la forma en que resuelven problemas, entre otros (Rodrigo, 1990). Dentro de dichos aspectos, la presente investigación se centró en la forma en que los niños que cursan primero básico manifiestan sus teorías sobre el mundo y sobre ellos mismos. La etapa preoperacional, faculta a estos niños de la función simbólica, la que se muestra a través de la imitación, el juego y el lenguaje (Santrock, 2006). Presentan un pensamiento de tipo animista, vale decir, tiende a "atribuir vida a objetos que no la tienen" y creer que "los objetos inanimados tienen la cualidad de estar vivos" (Papalia, 2001, p.372) y "ser capaces de actuar" (Santrock, 2006, p. 244). Poseen la "competencia de atribuir mente a otros y de predecir, y comprender su conducta en función de entidades mentales, tales como las creencias y deseos", también llamada teoría de la mente (Rivière y Núñez, 2001, p.10).

Ya en la década de los 30', Piaget (1997) se cuestionaba sobre las representaciones del mundo que se dan en forma espontánea en los niños. Planteamientos teóricos posteriores, sostienen que los niños desde muy temprana edad "se preocupan por el origen de las cosas, por el funcionamiento de la realidad y elaboran algún tipo de explicaciones para dar cuenta de estos asuntos" (Delval, 2001, p. 83), con independencia de que se les haya enseñado y hayan recibido instrucción formal sobre ello. Estas teorías se adquieren a través de la exploración regular del mundo y les permiten pensar los objetos, acontecimientos y personas que les rodean de un modo coherente como también predecir y controlar su realidad inmediata (Gardner, 1997; Pozo, 2000).

Algunos estudios realizados en Chile, ponen en evidencia la capacidad de los niños para dar explicaciones, elaborar y defender sus posturas. Junto con esto se destaca la importancia del entorno familiar en la construcción de las representaciones de los niños. Con respecto al papel de los educadores, se observó que sobrevaloran su propio conocimiento, inhibiendo la búsqueda de significados personales por parte de los niños (Bello \& Carrillo, 2007).

Otro estudio, sobre los argumentos infantiles frente a la transgresión de las normas, puso en evidencia que los niños menores consideran que transgredir las normas que imponen los adultos trae consecuencias negativas. Para estos niños, las normas tienen validez en cuanto permiten prevenir peligros y evitar daños (Barreiro, Kiper, \& Formoso, 2007).

El niño llega a la escuela con sus propias teorías, las que no siempre coinciden con las ideas de los adultos ni las propuestas por la ciencia, pero influyen considerablemente en su aprendizaje, intentando asimilar los conocimientos nuevos a sus aprendizajes anteriores y teorías implícitas a través de la búsqueda de elementos de información (Pozo, 2000).

Gardner (1997) señala que si hay correspondencia entre las formulaciones inventadas por el niño y las presentadas por los maestros, se origina una comprensión impor- tante en los niños, por lo que el propósito de la educación debiera ser revisar sus concepciones.

Asociado a los conceptos de "conocimientos previos" e "ideas previas", aparece el de Teorías subjetivas (TS), definidas por Groeben (1988) como "cogniciones de la visión de sí mismo y del mundo, que se pueden entender como un conjunto complejo, que tiene una estructura argumentativa, por lo menos implícita, y cumple las funciones de explicación, predicción y tecnología, contenidas también en las teorías científicas" (en Avendaño, Krause, \& Winkler, 1993, p.109). Son un tipo particular de creencias, que pueden contener conocimientos entre sus constituyentes. Estas explicaciones o teorías no científicas, son elaboradas por las personas para interpretar la realidad, orientar formas de acción y darle sentido a sus experiencias. Facilitan el desarrollo de guías para el comportamiento, es decir, permiten decidir entre diferentes alternativas conductuales. Las TS se manifiestan con una estructura y cierto grado de organización similar al de las hipótesis (Catalán, 2010).

En base a los antecedentes expuestos y considerando que las teorías de los niños impactan en su aprendizaje es relevante preguntarse:¿Cuáles y cómo son las teorías subjetivas que elaboran los niños de primero básico sobre sí mismo y sobre su entorno?

A partir de esta pregunta de investigación se formularon los siguientes objetivos. El Objetivo general: Analizar y comprender el desarrollo en la actividad explicativa sobre sí mismo y sobre su entorno de los niños que cursan primero básico en establecimientos educacionales de La Serena y Coquimbo para contribuir a la caracterización de su desarrollo cognitivo.

Para llevar a cabo dicho objetivo, se proponen los siguientes Objetivos específicos:1) Analizar la conducta y verbalizaciones de los niños en relación a su entorno y sobre ellos mismos que surgen en el contexto escolar; 2) Caracterizar e interpretar las teorías subjetivas de los niños acerca de sí mismos, 3) Caracterizar e interpretar las teorías subjetivas de los niños acerca de su entorno.

\section{Método}

Como diseño de investigación, se utilizó el estudio de caso de tipo intrínseco, debido a que la muestra estaba definida con anterioridad a que se implementara el estudio. Éste se realizó desde una aproximación metodológica de tipo cualitativa, para abordar los significados de los sujetos a través del estudio de sus teorías subjetivas.

Los participantes del estudio fueron niños, cuyas edades fluctuaron entre los 6 y los 9 años de edad, de ambos sexos, de nacionalidad chilena, pertenecientes a dos grupos de primer año de enseñanza básica de colegios de dependencia municipal de La Serena y Coquimbo, integrados por 27 y 23 alumnos, respectivamente. En todo momento se veló por resguardar la identidad de los alumnos y la confidencialidad en cuanto a la información recopilada. 
Durante las observaciones realizadas en el aula y patio se consideró a la totalidad del curso. En las entrevistas individuales de tipo semiestructuradas, participaron 4 alumnos por colegio, dos hombres y dos mujeres, cuyas edades fluctuaron entre los 6 y los 7 años. En las entrevistas de grupo focal, participaron 6 alumnos de cada colegio, 3 hombres y 3 mujeres, cuyas edades fluctuaron entre los 6 y los 7 años.

Para seleccionar a los alumnos que conformaron las entrevistas individuales y de grupo focal, se consideró la revisión de los registros de observación en aula y patios, procurando elegir a los alumnos que por sus intervenciones pudiesen aportar a la comprensión de las teorías subjetivas. Para confirmar la participación de los alumnos se pidió la autorización de los padres en forma oral y escrita.

La recolección de datos se realizó mediante tres procedimientos:

1) Observación: Se realizaron observaciones de 19 sesiones tanto dentro de las clases como fuera de ellas, por parte de una profesional educadora de párvulos. Complementariamente, a modo de triangulación, se realizaron observaciones en tres sesiones, por parte de una profesional psicóloga. En todos los casos, se llevó registro escrito de lo observado.

2) Entrevista semiestructurada: Se realizó una entrevista semiestructurada a 4 alumnos de cada colegio, con el fin de conocer con mayor detalle las explicaciones sobre algunos temas recurrentes dentro de la sala de clases y fuera de ella. Se elaboró un guion que orientó las preguntas de las entrevistas. El desarrollo de las entrevistas fue grabada en formato de audio y luego fueron transcritas y digitalizadas.

3) Entrevista de grupo focal: Se realizó una sesión de grupo focal para ampliar las explicaciones obtenidas por los métodos anteriormente descritos, a partir de las interacciones entre los participantes y para triangular los datos recopilados. Cada entrevista se grabó en formato de audio para su posterior transcripción.

El análisis de los datos recopilados se llevó a cabo mediante el procedimiento de la grounded theory, con apoyo del software Atlas Ti 5.0. Se implementaron dos de las tres codificaciones planteados por Strauss \& Corbin (2002): codificación abierta y codificación axial. A través de la codificación abierta, fue posible descomponer los textos transcritos e identificar categorías de análisis, que facilitaron la comprensión de la información recopilada en las observaciones y entrevistas. En segundo lugar, en la codificación axial, se reagruparon los datos que se fracturaron durante la codificación abierta, relacionando las categorías con sus subcategorías para formar explicaciones más precisas y completas sobre las teorías subjetivas encontradas.

\section{Resultados}

Mediante la codificación abierta se identificaron las TS que elaboran los niños en el contexto escolar, encontrándose que predomina la aparición de TS sobre el entorno y, con menor frecuencia TS sobre ellos mismos. Mediante la codificación axial se agruparon las categorías encontradas en subcategorías que se dan a conocer a continuación.

\section{TS sobre sí mismo}

Estas teorías aparecieron tanto dentro como fuera del aula, principalmente en la interacción con la observadora (Educadora de párvulos) y los compañeros. En la interacción con la investigadora (Psicóloga) las TS encontradas fueron intencionadas mediante las preguntas de la entrevista que se realizó en forma individual a los alumnos, donde se observó que un tema importante en su vida es la familia y las relaciones que establecen con sus miembros. También dieron explicaciones sobre sus actividades en el hogar, en su rutina diaria (como estudiar y jugar) como también sobre sus características físicas.

\section{TS sobre el entorno}

Se encontraron TS sobre el entorno con mayor frecuencia que las TS sobre sí mismos. Estas surgieron, tanto dentro como fuera del aula en la cotidianeidad de sus rutinas. Dentro del aula las TS surgen de forma espontánea, mayormente cuando los alumnos interactuaron con sus compañeros, en menor medida con la observadora y con la investigadora, y aún en menos oportunidades con la profesora. Con respecto a la interacción con los docentes, las TS encontradas se relacionan con las actividades y los contenidos revisados en clases. A continuación se da a conocer las principales subcategorías dentro de la categoría TS sobre el entorno:

\section{- Teorías sobre aspectos del colegio}

En general los alumnos manifiestan explicaciones positivas en torno al colegio, ya que les agrada asistir y perciben que estudiar es divertido. Dentro de sus explicaciones se encuentran aspectos sobre las personas con las que interactúan dentro del establecimiento.

\section{Las características de la observadora}

Los niños elaboran explicaciones en torno a lo que observan en las personas y en el ambiente. Así por ejemplo, en otros aspectos referidos a la persona de la observadora, explican la función de los frenillos: actúan en los dientes doblándolos hacia adentro y enderezándolos para que queden bien. Si las personas usan frenillos, entonces les crecerán los dientes derechos.

\section{La labor de la profesora}

Las explicaciones giran principalmente en torno al aspecto pedagógico y disciplinario: por un lado los niños reconocen que la profesora les enseña para ser más inteligentes, para aprender y pasar más rápido de curso. Por otro lado, 
reconocen que deben obedecerle para evitar sanciones y obtener consecuencias positivas (como por ejemplo, salir a recreo). También señalan que la docente interviene cuando hay problemas en el clima dentro del aula específicamente por el comportamiento disruptivo de sus compañeros, por lo que tratan de mantener una buena conducta para evitar que la profesora les llame la atención. Por otra parte, se observó que los alumnos consideraron las ideas de las docentes como un referente para adoptar sus ideas y transmitirlas a las demás personas, como se observa en la siguiente cita:

TS: V. C.: “iNo hay que respirar el viento helado' hace mal, dijo la profesora!" (Observación en aula 017)

\section{Las actividades de la clase}

Aprender- escribir- leer- estudiar: Los niños conciben que aprender es un fin en sí mismo dentro del colegio y consideran que es bueno escribir para obtener reconocimiento de sus familiares. Sobre la lectura, señalan que les agrada porque les permite aprender, acceder a los conocimientos por sí mismos, estudiar y pasar de curso. Los niños expresan que estudiar es un deber para ellos. Reconocen la importancia de estudiar para pasar de curso y no quedarse repitiendo. En el largo plazo, piensan que aprender los prepara para hacer las tareas en los distintos ámbitos del conocimiento. En el corto plazo, consideran que es importante trabajar en clases para así poder salir a recreo.

Pintar: Estas teorías fueron utilizadas por los niños para orientar sus acciones y las de sus pares, en situaciones donde no se contaba con los recursos específicos, como se muestra en la siguiente cita:

TS: “I. pinta los dibujos de su guía, toma sus lápices, moja la punta de uno con su lengua, luego pinta.

l.: "iTía si uno moja la punta del lápiz se hace acuarela!"”'(Observación en aula 010).

Del mismo modo, los niños se explican la mezcla de colores cuando les falta algún color de témpera (ejemplo: el blanco sirve para aclarar).

\section{- Teorías sobre los contenidos de la clase}

Jesús: Estas explicaciones ocurren a propósito de las actividades de la clase de religión, donde se señalan acontecimientos del origen, vida y muerte de este personaje; las cuales, en términos generales parecen ser reproducidas de lo aprendidas con sus padres y profesoras. En las entrevistas, los niños hacen atribuciones mágicas a la creación:

TS: “E: (...) ¿cómo Dios habrá creado la tierra y a la gente? $S$ : con su magia

E: ¿cómo hizo su magia? (...)
S: quería que no esté solo, quería amar, quería tener muchos hijos (...)

E: pero ¿cómo lo hizo, para hacer magia?

S: ah, no tenía más que hacer, le... con un poco de arena agarró e hicieron bolitas, hombres, animales con la arena (...) y cuando sopló les dio vida" (Entrevista Caso 3)

Reconocen que Jesús tiene emociones y que nuestros estados emocionales influyen en las suyas, lo que a su vez moviliza las acciones de Jesús en beneficio de nuestro bienestar. De igual modo como surgen explicaciones sobre Jesús, los alumnos se refieren a la figura del diablo.

El diablo: Los niños explican que el diablo era un ángel, que quería ser como Jesús pero no lo consiguió y se volvió malo, cambiando su apariencia física: aumentó su tamaño, le crecieron cuernos y su piel se volvió roja.Los niños piensan que el diablo existe debajo de la tierra, porque el sol es su debilidad y le puede provocar la muerte. En general, se explican las acciones de este personaje aludiendo a su maldad. Señalan que le gusta meterse dentro de las personas y matarlas, porque es malo. Creen que él hace realidad los sueños de las personas y después los mata y se los lleva al infierno. En general creen que las personas malas se van donde el diablo, cuando matan gente, roban o agreden a otros.

Los piratas, los dinosaurios y meteoritos: Estos temas aparecieron como resultado de la exposición de los alumnos frente al material preparado por la profesora para la clase, en este caso láminas que muestran objetos cuyo nombre comienza con las letras del abecedario. Por ejemplo, los piratas, personajes que según lo explicado por los niños, existieron hace años en Coquimbo, que buscan tesoros y nunca se cansan de hacerlo porque es una actividad que realizaban en forma recurrente. Piensan que viven en nunca jamás, que son caricaturas y no existen como tales en el presente.En cuanto a los dinosaurios, explican su desaparición de la tierra por la acción de un meteorito, específicamente por su explosión. Junto con esto, elaboran una TS de nivel teórico, con hipótesis más elaboradas (Catalán, 2010) donde caracterizan a los meteoritos y se refieren a sus funciones: Los meteoritos caen a la tierra, son de forma ovalada y de color amarillo, sirven para pedir deseos y para trasladar a los extraterrestres desde otro mundo hasta la tierra para enviarnos un mensaje. Señalan que luego de que el meteorito cayó a la tierra, hubo una explosión que la destruyó. Posteriormente comenzaron a existir los humanos.

\section{- Teorías implicadas en la relación con los pares}

Con respecto a los compañeros, identifican comportamientos inadecuados dentro del colegio como también las consecuencias negativas asociadas,como se observa en la siguiente cita:

TS: "C.: "iAh te voy a acusar!" 
M.: "¡Oye no me acusí, eso es ser acusete, después todos te van a molestar y te van a decir acusete!". (Observación en aula 022).

En ambos grupos se obtienen explicaciones sobre pelear, que difieren entre sí. En uno de ellos, los niños explican que pelear es una forma de defenderse en caso de que otras personas lo molesten:

TS: "S.: "iMi papá me enseñó a pelear!”

Observadora Fondecyt: “¿Con quién?”

S.: "iCon cualquiera que me moleste!"

Observadora Fondecyt: “¿Y cómo?”

S.: "¡Así mire, una pierna atrás, y la otra adelante, el brazo derecho atrás y el brazo izquierdo pegar combos, asi fuerte, y le pega combos y se muere al tiro!". (Observación en aula y patios 017)

En el otro grupo, reconocen que es importante no maltratar a los compañeros para evitar ser acusado con la profesora o el director, como también para evitar daños a la integridad física de sus compañeros. En el ámbito del trabajo en clases, se observó que los niños ayudaban a sus compañeros, motivándolos a cambiar la forma de ejecutar las tareas escolares por otras más precisas.

Amistad: Los niños señalan que los amigos juegan y ríen juntos. Pero no tan solo comparten buenos momentos, ya que pueden enojarse y junto con esto distanciarse, para luego volver a retomar su amistad. Perciben la relación de amistad como bastante flexible y reversible. En la relación de amistad distinguen como elementos clave la proximidad física, el cariño y la motivación por ser amigo de alguien. Esta relación implica además ayuda y amabilidad.

Los juegos: Los alumnos describen los juegos que realizan frecuentemente, como "La pillá" (pillada) y "La escondida", los que les divierten porque implica realizar una actividad física. Dentro del juego se aprecia que hay alumnos que asumen el liderazgo, los que determinan algunas normas para regular las interacciones:

TS: “J. le dice a Y.: “¿Puedo jugar?”

Y. le dice: “SSí, pero me dai chupete!"

J. hace un gesto de desgano.

Y. lo mira y le dice: "iSi no, no, no jugai!" se aleja corriendo. (...)

Un niño que juega a la pillada le dice a Y.: "¡Oye, oye! ¿Puede jugar el A.?”
Y.: "No, yo mando el juego y tú no jugai!" (Observación en aula 028)

\section{- Teorías sobre aspectos del hogar}

La familia: Los alumnos explican la relación con los miembros del grupo familiar, la percepción de sus características psicológicas, sus emociones y las actividades que realizan. Las TS de los niños sobre los miembros de la familia variaron en función del tipo y cualidad de las relaciones que mantienen con estas personas.

\section{- Otras teorías}

Dentro de los diálogos que se desarrollaron por los alumnos, surgieron temas específicos frente a los cuales manifestaron sus explicaciones:

\section{* Aspectos de la vida y de la muerte}

La existencia: Los niños consideran que la existencia está marcada por la vida: si algo está vivo existe y si algo está muerto entonces no existe.

La muerte: Los niños consideran que cuando las personas mueren, su alma sale del cuerpo y desaparece de la tierra: si son buenas se van al cielo y si son malos al infierno. Dentro de las causas de la muerte reconocen algunas dependientes de factores internos como el envejecimiento $y$ las enfermedades, y otras dependientes de factores externos, como los asesinatos.

\section{* Teorías sobre insumos provenientes de los animales y vegetales:}

La lana: Los niños fundamentan que la vestimenta de lana abriga:

"L: la lana da calor porque se acumula mucho y como es de laaa...oveja se le junta y el calor de la oveja le da el calor a la lana después la hecha en una maquinita la lana la deja, la tira asi”" (Entrevista Caso 4)

La leche: Señalan que la leche contribuye a su crecimiento y a mantener una buena salud. Consumirla es bueno porque tiene calcio y este elemento ayuda a los huesos a crecer como también para los dientes porque es muy nutritiva.

El tomate: Explican las condiciones para su consumo: Si el tomate está verde, entonces no está maduro por lo que todavía no es para comérselo, ya que hace mal para el estómago. Por el contrario, cuando el tomate está rojo lo podemos comer, porque ya maduró y no hace mal para el estómago.

\section{* Teorías sobre seres mitológicos}

La Llorona: Los niños sostienen que esta personaje de leyenda no existe, porque está muerta. Otros señalan que esta mujer estuvo muerta pero se transformó en la Llo- 
rona, porque cuando las personas mueren el alma se revive y se transforma en un fantasma que asusta a las personas. Consideran que hay fantasmas malos y fantasmas buenos: los buenos son de Dios y los malos son del Diablo. La Llorona es un fantasma del diablo.Otros niños creen que existe, porque la han visto tanto en películas como en la realidad y que llora todos los días porque se le murieron sus hijos. Desde entonces se dedica a destruir casas, asustar y a matar a las personas.

\section{* Teorías sobre elementos de la comunidad}

Las alarmas: Al escuchar el sonido de una alarma los niños predicen la ocurrencia de robos o situaciones perjudiciales para las personas, lo que los moviliza a la acción de comprobar por ellos mismos esta teoría, observando hacia los lugares desde donde proviene el sonido.

Los bomberos: Los niños reconocen una misión social en la labor de los bomberos que es salvar personas y evitar consecuencias negativas en su vida. Su misión la llevan a cabo, gracias a las propiedades del agua:

TS: "L: porque el agua es fría y el fuego es caliente y como el frio siempre gana, le echa mucho así o la deja mucho rato y la manguera siente que se quema

E: ¿por qué el frío siempre gana?

L: porque el frío es como que lo congela" (Entrevista Caso 4)

\section{Discusión}

Los resultados de la presente investigación ponen en evidencia que en las verbalizaciones de los niños aparecen descripciones, comparaciones, relaciones, predicciones, inferencias, descubrimientos, argumentaciones, explicaciones y teorías sobre ellos mismos y su entorno para operar en su vida, lo que concuerda con lo establecido en la literatura sobre el desarrollo cognitivo de los niños (Delval, 2001; Gardner, 1997; Pozo, 2000).

En cuanto al rol docente, el comportamiento mostrado en experiencias anteriores fue un elemento importante en las teorías de los niños porque éstos actuaron en función de las conductas que consideraron adecuadas para el juicio de los docentes, para lograr su aprobación y evitar sanciones. En este punto se evidencia la moralidad heterónoma correspondiente al nivel preconvencional propuesto por Kohlberg (Papalia 2001), donde los niños actúan correctamente para evitar el castigo. Hallazgos similares son los propuestos por Barreiro, Kiper, \& Formoso (2007), quienes encontraron que los niños de esta edad consideran que transgredir las normas que imponen los adultos trae consecuencias negativas.Se observó que el rol de los docentes en la clase actuó facilitando, como también inhibiendo la participación de los alumnos. Dentro de los elementos facilitadores, se encontró que los recursos de enseñanza y las actividades propuestos en clases permitieron que los niños verbalizaran algunas explicaciones y descubrimientos. Dentro de los elementos que inhibidores de la actividad explicativa de los niños, los intentos reiterados de los educadores por mantener la disciplina dentro del aula.

Se observó que los alumnos elaboran TS, pero se podría haber provocado una actividad reflexiva más profunda, para obtener explicaciones más extensas. Estos hallazgos también se condicen con lo encontrado por Bello \& Carrillo (2007), quienes observaron que los educadores inhibieron la búsqueda de significados personales por parte de los niños.

Con respecto a sus pares, se encontró que los niños explican y justifican tanto las verbalizaciones como los comportamientos de sus compañeros, como también predicen sus conductas futuras. En estas explicaciones hay indicios de teoría de la mente, es decir, que son capaces de atribuir estados mentales en las demás personas (Santrock, 2006; Papalia, 2001; Rivière, \& Núñez, 2001). Los niños expresaron sus propias concepciones morales sobre lo que se debe y no debe hacer, para cuestionar y también darle sentido a la conducta de sus compañeros, lo que concuerda con los aspectos señalados por Santrock (2006) en torno al desarrollo moral de los niños. Los niños trataron de persuadir a sus pares (con relativo éxito), en base a sus explicaciones, de que su pensamiento es el correcto y, por tanto influir en su conducta indicándoles cómo deben actuar.

Dentro de las interacciones con sus pares, la situación de juego fue un aspecto importante en la rutina de los niños donde surgieron explicaciones. En el juego se expresa la función simbólica, atributo esencial del niño en la etapa preoperacional que define Piaget (Papalia, 2001).De esta misma manera se observan elementos característicos del pensamiento infantil preoperacional como el egocentrismo y animismo. Por una parte, manifiestan dificultad para distinguir entre la perspectiva propia y la de otras personas como también atribuyen ccaracterísticas de los seres vivos a los objetos (Santrock, 2006; Papalia, 2001).

Bello \& Carrillo (2007) destacaron la importancia del entorno familiar en la construcción de las representaciones de los niños, situación que también fue observada en el presente estudio. El rol de la familia fue importante en el desarrollo de la actividad explicativa de los niños, en cuanto les transmiten sus propias explicaciones sobre el mundo, orientándoles a la acción en determinadas situaciones, como por ejemplo emplear conductas de defensa frente a las agresiones de sus pares.

En relación a las TS como tales, se encontraron enunciados con una estructura formal asimilable consistentemente a la de una hipótesis, condición básica para la existencia de una teoría. Estos enunciados fueron predominantemente de escasa elaboración para ambos colegios. En relación al significado emocional se encontraron con mayor frecuencia TS de tipo positivas, que manifiestan una mayor cercanía emocional con los objetos de teorización que aparecieron en los dos casos estudiados. Con respecto a la orientación explicativa, se hallaron tanto TS de tipo regresivas como progresivas, encontrándose enunciados que permitían ex- 
plicar, justificar como también predecir situaciones. Sobre la orientación a la acción se encontraron TS en mayor medida de tipo iniciadoras de acción, lo que evidencia la motivación y energía que gatilla el inicio de las acciones infantiles. A modo de conclusión, se puede afirmar que los niños construyen TS, que las expresan en forma poco elaborada, pero que si se les incentiva a la reflexión pueden dar respuestas de mayor complejidad; lo que hace relevante el promover la reflexión, especialmente en la actividad pedagógica en el aula. En general, los datos provenientes de las observaciones evidencian fundamentalmente esbozos de teoría y la identificación de temáticas del interés general de los niños, sobre las que éstos pueden desarrollar explicaciones. Tanto las entrevistas individuales y el grupo focal, permitieron desplegar explicaciones, confirmando la existencia de éstas como también la potencialidad del recurso metodológico empleado para acceder a las teorías subjetivas.

Se encontró que para los dos casos estudiados los niños elaboran predominantemente teorías sobre elementos de su entorno. Los niños construyen sus teorías basándose en sus experiencias previas y las utilizan en gran medida para predecir y explicar las situaciones, persuadir a las demás personas como también orientar sus propias acciones futuras, mostrando además una tendencia a corroborar y probar sus teorías en el ambiente. Se observan escasos hallazgos en cuanto a las TS que tienen los alumnos sobre ellos mismos. Este hecho se convierte en un fundamento para realizar futuros estudios al respecto de las TS infantiles, campo poco abordado en el ámbito de la psicología, particularmente desde una perspectiva de estudio cualitativa.

Algunos de los hallazgos encontrados coindicen con los de otros estudios realizados con anterioridad en otras regiones del país, por lo que se puede conjeturar que la actividad reflexiva de los niños de un mismo grupo etario tiene un desarrollo similar independiente del espacio geográfico o ambiente donde se desarrolle.

\section{Referencias}

Avendaño, C., Krause, M., \& Winkler, M. (1993). Representaciones sociales y teorías subjetivas: relevancia teórica y aplicaciones empíricas. Revista Psykhe, 1, 107-114.

Barreiro, A., Kiper, C., \& Formoso, J. (2007). Transgresión de las normas: análisis de argumentos infantiles. Perspectivas en Psicología: Revista de Psicología y Ciencias afines, 4, 38-43.
Bello, L. \& Carrillo, C. (2007). Cosmovisión infantil; creencias de niños preescolares de la Región de Magallanes. Punta Arenas, Universidad de Magallanes. Tesis para optar al Título de Educadora de Párvulos. Recuperado: 17. Sep. 2013. Disponível: www.umag. cl/biblioteca/tesis/bello_lorena_2007.pdf

Catalán, J. (2010). Teorías subjetivas aspectos teóricos y prácticos. La Serena: ULS Editorial.

Delval, J. (2001). Descubrir el pensamiento de los niños. Barcelona: Paidós.

Gardner, H. (1997). La mente no escolarizada. Cómo piensan los niños y cómo deberían enseñar las escuelas. Buenos Aires: Paidós.

Papalia, D. (2001). Psicología del desarrollo. Bogotá: Mc Graw Hill.

Piaget, J. (1997). La representación del mundo en el niño. Madrid: Ediciones Morata.

Pozo, J. (2000). Aprendices y maestros: La nueva cultura del aprendizaje. Madrid: Alianza Editorial.

Rivière, A. \& Núñez, M. (2001). La mirada mental. Buenos Aires: Editorial Aike.

Rodrigo, M. J. (1990). Procesos cognitivos básicos años preescolares. En J. Palacios, A. Marchesi, \& C. Coll, (Comp.), Desarrollo psicológico y educación, I. (pp. 143-155). Madrid: Alianza Psicología.

Santrock, J. (2006). Psicología del desarrollo. El ciclo vital. Madrid: Mc Graw Hill.

Strauss, A., \& Corbin, J, (2002): Bases de la investigación cualitativa. Técnicas y procedimientos para desarrollar la teoría fundamentada. Antioquia: Editorial Universidad de Antioquia. Primera Edición en Español. 
Sobre os autores

Lisette Gahona Palominos (ps.lisettegp@gmail.com)

Universidad de La Serena, Chile

Jorge Catalán Ahumada (jcatalan@userena.cl)

Universidad de La Serena, Chile 